\title{
Metformin Inhibits Advanced Glycation End Products- Induced Cell Apoptosis and Oxidative Stress of Human Skin Fibroblasts by Downregulating MicroRNA-126
}

\author{
C. X. WANG, J.ZHANG ${ }^{1}$,YANYAN LI, S. WEN, CONGCONG WANG, CHENGLIN XU, YANJU HE AND L. ZHOU*
}

Department of Endocrinology, Shanghai Pudong Hospital, Fudan University Pudong Medical Center, Shanghai 201399, ${ }^{1}$ Department of Endocrinology and Metabology, The First Affiliated Hospital of Shandong First Medical University \& Shandong Provincial Qianfoshan Hospital, Shandong Key Laboratory of Rheumatic Disease and Translational Medicine, Shandong Institute of Nephrology, Shandong 250014, China

\section{Wang et al.: Metformin Inhibits Advanced Glycation End Products-Induced Cell Apoptosis}

\begin{abstract}
To determine the influence of metformin on the proliferation and apoptosis of advanced glycation end products-induced primary skin fibroblasts and its mechanism. Human skin fibroblasts were purchased and treated with advanced glycation end products of different concentrations separately, on which the fibroblasts were intervened by metformin of different concentrations. The proliferation and apoptosis of cells treated with advanced glycation end products and metformin were evaluated and the changes of oxidative stress markers and microRNA-126 in the cells were also determined. In addition, the cells treated with metformin were transfected with miR-126 mimics and blank plasmids and microRNA-126 mimics, separately, to explore whether the transfection can weaken the effects of metformin. Human skin fibroblasts lines HSF2 showed a decrease in proliferation and superoxide dismutase and an increase in apoptosis rate, reactive oxygen species and malondialdehyde after being treated with advanced glycation end products and metformin weakened the influence of advanced glycation end products on proliferation, apoptosis as well as oxidative stress of human skin fibroblasts cells. In addition, HSF2 cells showed increased microRNA-126 expression after being treated with advanced glycation end products and metformin could inhibit this increase. Transfection of microRNA-126 mimics weakened the protective effect of metformin on HSF2 cells treated with advanced glycation end products. Metformin can inhibit advanced glycation end products-induced cell apoptosis and oxidative stress of human skin fibroblasts by downregulating microRNA-126.
\end{abstract}

Key words: Metformin, microRNA-126, skin fibroblasts, advanced glycation end products

Diabetes is a common metabolic disease, burdening human life, health and medical expenses ${ }^{[1]}$. It is estimated that there were 451 million patients with Diabetes Mellitus (DM) worldwide in 2017 and the number would be 693 million by $2045^{[2]}$. Patients with DM usually suffer from various complications of which diabetic foot ulcer is considered to be one of the most serious complications ${ }^{[3]}$. Although a great progress has been achieved in the overall clinical medicine over the past few decades, there is still no effective treatment for diabetic foot ulcer widely used ${ }^{[4]}$. Metformin (MET) is a derivative of biguanides and a first-line drug for the treatment of type $2 \mathrm{DM}^{[5]}$. Many evidences have verified that MET not only has good hypoglycemic effect, but also can promote diabetic wound healing ${ }^{[6,7]}$, but the mechanism is still under investigation.

MicroRNA (miRNA) is a highly conserved non-coding small RNA, 18-25 nucleotides in length, which is widely found in animals and plants. It can affect cell differentiation, proliferation, metabolism as well as apoptosis by suppressing the transcription of proteincoding genes or by accelerating messenger RNA (mRNA) degradation of them ${ }^{[8]}$. With the deepening understanding of microRNA (miR), it is acquired that $\mathrm{miR}$ is a crucial participant in skin growth ${ }^{[9]}$. Earlier studies have revealed that MET can regulate the basic biological behavior of cells through regulating miR, thus affecting disease development. For example, MET can inhibit the growth of renal cancer cells by increasing miR-26a ${ }^{[10]}$ and it can inhibit the proliferation of liver cancer cells and induce their cycle arrest by regulating miR-378 ${ }^{[11]}$. In addition, MET can also ameliorate skeletal muscle insulin resistance through suppressing miR-21 expression ${ }^{[12]}$. As a widely studied miR, miR126 is found to be elevated in patients with type 2 DM, while its expression is down-regulated after MET

*Address for correspondence

E-mail: zhouligang@yahoo.com 
treatment ${ }^{[13,14]}$. Poor diabetic wound healing is the result of a combination of many factors and the decrease of proliferation of fibroblasts and the increase of apoptosis of them are key factors ${ }^{[15]}$. In addition, according to previous studies, miR-126 can regulate the proliferation and apoptosis of fibroblasts ${ }^{[16,17]}$. We suspected that the effect of MET on diabetic wound healing may be related to the regulation of $\mathrm{miR}-126$ on the proliferation and apoptosis of fibroblasts.

In our study, we mainly analyzed the influence of MET on the proliferation and apoptosis of Advanced Glycation End products (AGEs)-induced primary skin fibroblasts and the relationship between MET and miR126.

\section{MATERIALS AND METHODS}

\section{Cell source and culture:}

Human skin fibroblast lines (HSF2) purchased from Shanghai SIMR Biotechnology Company were incubated in dulbecco's modified eagle medium (DMEM) supplemented with $15 \%$ fetal bovine serum (FBS; Gibco, USA), 100 U/ml penicillin (Gibco, USA), as well as $100 \mathrm{U} / \mathrm{ml}$ streptomycin (Gibco, USA) in a 5 $\% \mathrm{CO}_{2}$ incubator at $37^{\circ}$ and then the cells were passaged when they reached $80 \%-90 \%$ confluency after they attached to the wall and sprawled out.

\section{Cell processing:}

Treatment with AGEs (BioVision, USA): HSF2 cells in logarithmic growth stage were taken and AGEs was diluted with complete culture medium to 100,200 and $300 \mu \mathrm{g} / \mathrm{ml}$, separately. A 16 well plate was added with cell culture solution containing different concentrations at $100 \mu \mathrm{l} /$ well and the control group was added with only $100 \mu \mathrm{l}$ complete culture medium.

Treatment with MET (Sigma, USA): Cells were added with $100 \mu \mathrm{l} \mathrm{MET} \mathrm{at} \mathrm{different} \mathrm{concentrations} \mathrm{(5,}$ 10 and $20 \mathrm{mmol} / \mathrm{l}$ ) separately, after being treated with $300 \mu \mathrm{g} / \mathrm{ml}$ AGEs, while the control group was added with only $100 \mu 1$ complete medium.

Cell transfection: MiR-126 mimics and blank plasmids (miR-NC) were constructed with plasmid cloning DNA (pcDNA) 3.1 plasmids as vectors, separately and they were transfected into HSF2 cells treated by MET with Lipofectamine $^{\mathrm{TM}} 2000$ reagent (Invitrogen, USA) under reagent instructions, followed by $2 \mathrm{~h}$ incubation.

\section{Quantitative reverse transcription polymerase chain reaction ( $\mathrm{qRT}-\mathrm{PCR})$ assay:}

Total RNA was obtained from cells through a TRIzol kit (Invitrogen Company, USA) and its concentration, purity, as well as integrity were determined using an ultraviolet spectrophotometer and agarose gel electrophoresis. Afterwards, reverse transcription was performed on the total RNA using a reverse transcription kit (Invitrogen, USA) and amplification was performed through SYBR_Premix ExTaq II (Takara, Dalian, China) under the amplification system consisting of 20 $\mu l$ total reaction volume with $10 \mu \mathrm{l}$ SYBR Premix Ex Taq II (2X), $2 \mu$ l complementary DNA (cDNA), $0.8 \mu \mathrm{l}$ upstream primer, $0.8 \mu \mathrm{l}$ downstream primer, as well as sterile purified water added to adjust the volume. The amplification conditions: $95^{\circ}$ for $30 \mathrm{~s}$, followed by 40 cycles of $95^{\circ}$ for $5 \mathrm{~s}$ and $60^{\circ}$ for $30 \mathrm{~s}$. Data in this study were analyzed through $2^{-\Delta \Delta \mathrm{ct}[18]}$.

\section{Proliferation determination by Cell Counting kit-8 (CCK-8) assay:}

Treated cells were harvested and prepared into $1 \times 10^{5}$ cells $/ \mathrm{ml}$ cell suspension and the suspension was transferred to a 96 well plate. CCK-8 solution (10 $\mu \mathrm{l}$; Dojindo, Japan) was put in each well at $24 \mathrm{~h}$ after culturing, followed by $2 \mathrm{~h}$ incubation with dark surroundings. Finally, the optical density of cells in every well at $490 \mathrm{~nm}$ was determined through a microplate reader (Molecular Devices, USA) and corresponding growth curves were drawn.

\section{Cell apoptosis determination:}

Treated cells were harvested and trypsinized through $0.25 \%$ trypsin and then prepared into $1 \times 10^{6}$ cells/ $\mathrm{ml}$ suspension. The suspension was put with Annexin V-FITC/propidium iodide (PI) (MultiSciences (Lianke) Biotech Co., Ltd., Hangzhou, China) in order, incubated at indoor temperature with dark surroundings for $5 \mathrm{~min}$ and finally the apoptosis of the cells was detected using a flow cytometer.

\section{Determination of oxidative stress markers in cells:}

The cells to be determined were collected and digested with trypsin and then centrifuged to discard the supernatant. Subsequently, the cells were added with Phosphate Buffered Saline (PBS) to resuspend the cells and then the cells were crushed by an ultrasonic cell crusher, followed by $3500 \times \mathrm{g}$ centrifugation for 10 min to collect the supernatant. Finally, the activities and levels of Malondialdehyde (MDA), Superoxide Dismutase (SOD) and Catalase (CAT) in the supernatant were quantified using corresponding MDA, SOD and CAT kits (all purchased from Nanjing Jiancheng Bioengineering Institute) according to kit instructions. 


\section{Statistical analyses:}

In our study, the acquired data were processed statistically through Statistical Package For The Social Sciences (SPSS) 1.8.0 and visualized into figures through GraphPad 7. Inter-group comparison was carried out through the independent $t$ test and multigroup comparison was carried out through the one-way Analysis Of Variance (ANOVA). Post hoc pairwise comparison was carried out using the Least Significant Difference (LSD) t test and comparison of expression at different time points was carried out through the repeated measures analysis of variance. Bonferroni post hoc test was also adopted, $\mathrm{p}<0.05$ implies a remarkable difference.

\section{RESULTS AND DISCUSSION}

Effects of AGEs on proliferation, apoptosis and oxidative stress of HSF2 cells were determined. Determination of the proliferation and apoptosis of HSF2 cells by CCK8 and flow cytometry revealed that after being treated with AGEs, HSF2 cells showed inhibited proliferation ability, increased apoptosis rate and a concentration dependence and determination of oxidative stress markers in HSF2 cells treated with AGEs revealed that AGEs could increase the MDA level in HSF2 cells and reduce SOD and CAT levels in them and HSF2 cells showed a concentration dependence of AGEs (fig. 1).

MET could weaken the effects of AGEs on the proliferation, apoptosis and oxidative stress of HSF2 cells. For the purpose of determining the influence of MET on AGEs-induced HSF2 cells, we treated HSF2 cells with different concentrations of MET after treating them with $300 \mu \mathrm{g} / \mathrm{ml}$ AGEs. Evaluation of the proliferation and apoptosis of treated cells revealed that MET could weaken the influence of AGEs on the proliferation and apoptosis of HSF2 cells and the weakening effect would be enhanced with the increase of MET concentration. Subsequently, we quantified oxidative stress markers in cells in each group, finding that MET could also effectively weaken the effects of AGEs on the levels of CAT, MDA and SOD in HSF2 cells (fig. 2).

AGEs increased the miR-126 expression in HSF2 cells and MET could inhibit this effect. In order to explore the ability of MET in inhibiting the biological behavior changes of HSF2 cells induced by AGEs, we quantified miR-126 in HSF2 cells treated by AGEs and MET. It came out that AGEs increased the miR-126 expression in HSF 2 cells in a concentration-dependent manner but MET could inhibit the increase of miR-126 expression in HSF2 cells caused by AGEs (fig. 3).

The increase of miR-126 could weaken the therapeutic effect of MET. According to the above results, MET may reduce the damage of AGEs to HSF2 cells by regulating miR-126. For the purpose of confirming the relationship between MET and miR-126, we treated HSF2 cells with MET and transfected them with miR$\mathrm{NC}$ and miR-126 mimics and then observed the changes of cell biological behaviors, finding that in contrast to the MET + miR-NC group, the MET + miR-126 mimics group showed increased miR-126 expression, inhibited cell proliferation and intensified cell apoptosis. We also quantified oxidative stress markers in each group, finding that in contrast to the MET + miR-NC group, the $\mathrm{MET}+$ miR-126 mimics and MET+miR-126 mimics groups showed increased CAT and MDA levels and decreased SOD level (fig. 4).

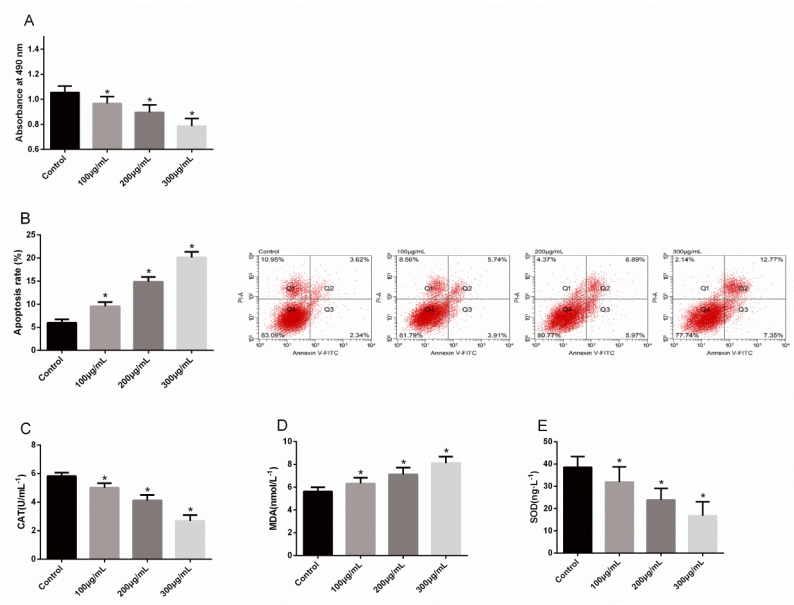

Fig. 1: Effects of AGEs on the proliferation, apoptosis and oxidative stress of HSF2 cells. (A) The proliferation ability of HSF2 cells was inhibited after treatment with AGEs; (B) The apoptosis rate of HSF2 cells was increased after treatment with AGEs (C) The CAT level in HSF2 cells decreased after treatment with AGEs; (D) The MDA level in HSF2 cells increased after treatment with AGEs; (E) The SOD level in HSF2 cells decreased after treatment with AGEs Note: *indicates $\mathbf{p}<0.05$ vs. the control group 

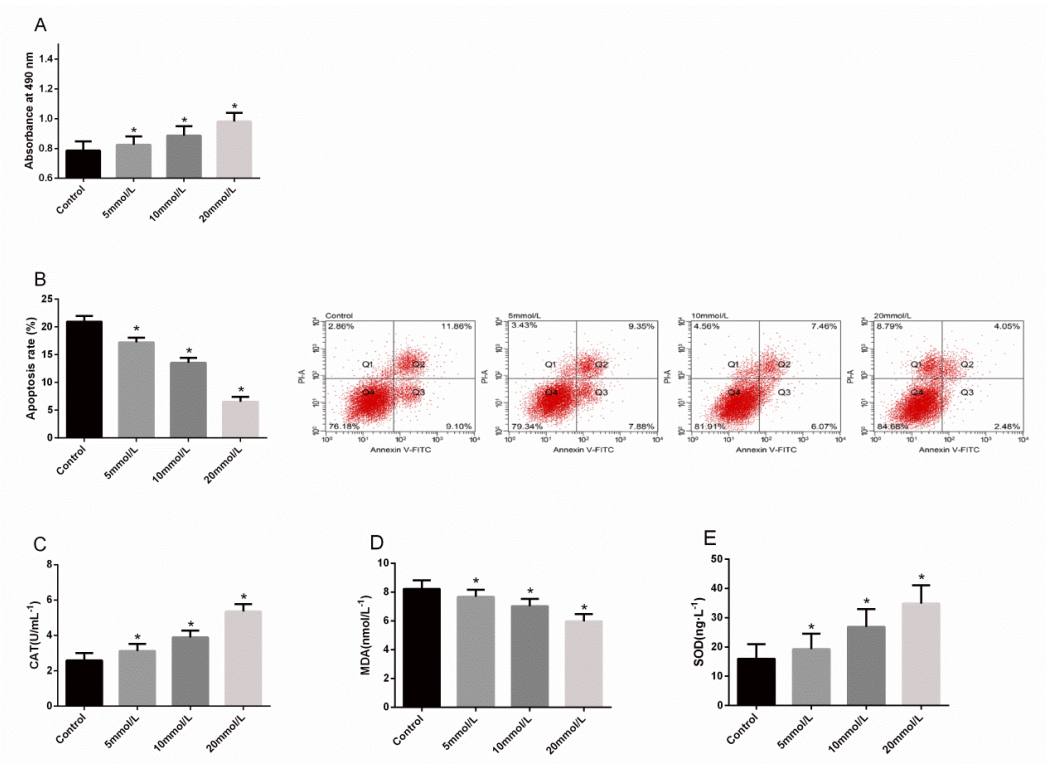

Fig. 2: MET could weaken the effects of AGEs on the proliferation, apoptosis and oxidative stress of HSF2 cells. (A) MET could weaken the effects of AGEs on the proliferation of HSF2 cells; (B) MET could weaken the effects of AGEs on the apoptosis of HSF2 cells; (C) MET could weaken the effects of AGEs on the CAT level in HSF2 cells (D) MET could weaken the effects of AGEs on the MDA level in HSF2 cells; (E) MET could weaken the effects of AGEs on the SOD level in HSF2 cells Note: *indicates $\mathbf{p}<0.05$ vs. the control group
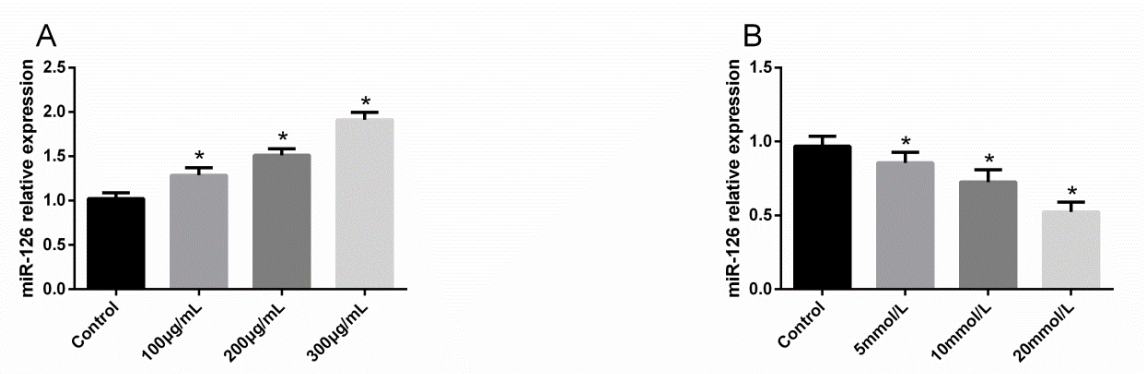

Fig. 3: AGEs increased the miR-126 expression in HSF2 cells and MET could inhibit this effect. (A) AGEs increased the miR126 expression in HSF2 cells; (B) MET weakened the effects of AGEs on the apoptosis of HSF2 cells Note: * indicates $\mathbf{p}<0.05$ vs. the control group
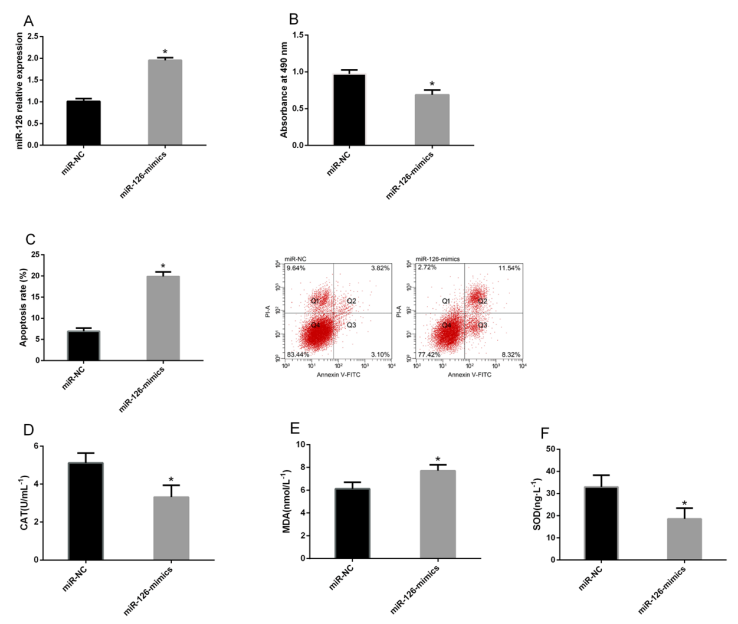

Fig. 4: The increase of miR-126 could weaken the therapeutic effect of MET. (A) Compared with the MET+miR-NC group, the MET+miR-126 mimics group showed up-regulated miR-126; (B) Compared with the MET + miR-NC group, the MET + miR-126 mimics group showed decreased cell proliferation; (C) Compared with the MET + miR-NC group, the MET+miR-126 mimics group showed increased apoptosis; (D) Compared with the MET+miR-NC group, the MET+miR-126 mimics group showed decreased CAT level; (E) Compared with the MET+miR-NC group, the MET+miR-126 mimics group showed increased MDA level; (F) Compared with the MET+miR-NC group, the MET+miR-126 mimics group showed decreased SOD level Note: *indicates $\mathbf{p}<0.05$ vs. the MET+miR-NC group 
About one quarter of patients with DM suffer from foot ulcers at some point in their lives and despite drug treatment, more than two thirds of the patients will suffer from unhealed wounds ${ }^{[19,20]}$. At present, the pathogenesis of diabetic skin lesions is still unclear and exploring its mechanism has crucial clinical value for improving the prognosis of patients. In our study, we mainly analyzed the influence of MET on the proliferation and apoptosis of AGEs-induced skin fibroblasts and the relationship between MET and miR-126, finding that MET inhibited AGEs-induced apoptosis and oxidative stress of human skin fibroblasts by downregulating miR-126.

AGEs are harmful metabolites, which have been found to accumulate in large quantities in patients with DM compared with healthy individuals ${ }^{[21]}$. One previous study has revealed that AGEs are a primary cause of skin fibroblast death in patients with $\mathrm{DM}^{[22]}$. Fibroblasts are the main cells that synthesize and secrete collagen and fibronectin play a key role in wound healing. Changes in the function of skin fibroblasts are one of the main reasons for poor wound healing in patients with $\mathrm{DM}^{[15]}$. In this study, we found that after being treated with AGEs, HSF2 cells showed inhibited proliferation ability, increased apoptosis rate and a concentration dependence. AGEs can induce the production of reactive oxygen species in cells and cause oxidative stress, thus damaging skin fibroblasts ${ }^{[23,24]}$. This study found that after treatment with AGEs, the MDA level in skin fibroblasts increased, while the SOD and CAT levels in them decreased, which implied that AGEs are one of the important killers that lead to unnatural apoptosis and oxidative stress damage of fibroblasts. One earlier study has revealed that MET can reduce the formation and accumulation of AGEs in human body ${ }^{[25]}$, which suggests that MET can protect skin fibroblasts from AGEs. Our results also verified that MET can weaken the effects of AGEs on the function and oxidative stress of skin fibroblasts.

To explore how MET protects skin fibroblasts from AGEs, we analyzed the correlation of miR-126 with MET and AGEs. One previous study has pointed out that miR-126 takes a crucial part in the development of various diseases. In our study, we determined the changes of miR-126 in skin fibroblasts treated by AGEs and found that miR-126 expression increased after treatment with AGEs and MET inhibited the increase, indicating that MET may protect skin fibroblasts from AGEs by regulating miR-126. In order to further understand the correlation between MET and miR126, we treated AGEs-induced skin fibroblasts with
MET and transfected them with miR-NC and miR-126 mimics, separately, finding that increasing miR-126 could weaken the effects of MET on skin fibroblasts after AGEs induction, which implied that MET could inhibit AGEs-induced apoptosis and oxidative stress of human skin fibroblasts by downregulating miR-126.

This study has verified that MET can inhibit AGEsinduced apoptosis and oxidative stress of skin fibroblasts and it may be related to the regulation of MET on miR-126. We reviewed previous researches on the role of miR-126 in human diseases and found that miR-126 can participate in the progression of diseases by activating or inhibiting some common pathways, such as Phosphatidy Linositol 3-kinase/protein kinase B (PI3K/Akt) and Ras homolog family member A(RhoA)/ Rho-associated protein kinase (ROCK) (RhoA/ROCK) [26,27]. Therefore, we suspected that MET can affect the biological behavior of human skin fibroblasts through regulating miR-126 and mediating related pathways, which can be extensively studied in the future to further explore the potential mechanism of MET in promoting wound healing in patients with DM.

To sum up, MET inhibits AGEs-induced apoptosis and oxidative stress of human skin fibroblasts by downregulating miR-126.

\section{Funding:}

This work was supported by National Natural Science Foundation of China (81370932), the Key Studies (Special) Department Fund of the Pudong, New Area Health Planning Commission (PWZzk2017-03) and Integrative Medicine special fund of Shanghai Municipal Health Planning Committee (ZHYYZXYJHZX-2-201712), Outstanding Leaders Training Program of Pudong Health Bureau of Shanghai (PWR12014-06), the Outstanding Clinical Discipline Project of Shanghai Pudong (PWYgy-2018-08), the Natural Science Foundation of China (Project no. 21675034), Shanghai Natural Science Foundation (19ZR1447500).

\section{Conflicts of interest:}

The authors declared no conflict of interest.

\section{REFERENCES}

1. Kido D, Mizutani K, Takeda K, Mikami R, Matsuura T, Iwasaki $\mathrm{K}$, et al. Impact of diabetes on gingival wound healing via oxidative stress. PLoS One 2017;12(12):e0189601.

2. Cho N, Shaw JE, Karuranga S, Huang YD, da Rocha Fernandes JD, Ohlrogge AW, et al. IDF Diabetes Atlas: Global estimates of diabetes prevalence for 2017 and projections for 2045 . Diabetes Res Clin Pract 2018;138:271-81. 
3. Guo Y, Lin $\mathrm{C}, \mathrm{Xu} \mathrm{P}, \mathrm{Wu} \mathrm{S}, \mathrm{Fu} \mathrm{X}, \mathrm{Xia} \mathrm{W}$, et al. AGEs induced autophagy impairs cutaneous wound healing via stimulating macrophage polarization to $\mathrm{M} 1$ in diabetes. Sci Rep 2016;6(1):1-6.

4. Xiao J, Chen S, Yi J, Zhang HF, Ameer GA. A cooperative copper metal-organic framework-hydrogel system improves wound healing in diabetes. Adv Funct Mater 2017;27(1):1604872.

5. Inzucchi SE, Bergenstal RM, Buse JB, Diamant M, Ferrannini E, Nauck M, et al. Management of hyperglycemia in type 2 diabetes, 2015: a patient-centered approach: update to a position statement of the American Diabetes Association and the European Association for the Study of Diabetes. Diabetes Care 2015;38(1):140-9.

6. Han X, Tao Y, Deng Y, Yu J, Sun Y, Jiang G. Metformin accelerates wound healing in type 2 diabetic $\mathrm{db} / \mathrm{db}$ mice. Mol Med Rep 2017;16(6):8691-8.

7. Inouye KA, Bisch FC, Elsalanty ME, Zakhary I, Khashaba RM, Borke JL. Effect of metformin on periimplant wound healing in a rat model of type 2 diabetes. Implant Dent 2014;23(3):319-27.

8. Fabian MR, Sundermeier TR, Sonenberg N. Understanding how miRNAs post-transcriptionally regulate gene expression. Prog Mol Subcell Biol 2010;50:1-20.

9. Schneider MR. MicroRNAs as novel players in skin development, homeostasis and disease. $\mathrm{Br} \mathrm{J}$ Dermatol 2012;166(1):22-8.

10. Yang FQ, Wang JJ, Yan JS, Huang JH, Li W, Che JP, et al. Metformin inhibits cell growth by upregulating microRNA26a in renal cancer cells. Int J Clin Exp Med 2014;7(10):3289.

11. Zhou J, Han S, Qian W, Gu Y, Li X, Yang K. Metformin induces miR-378 to downregulate the CDK1, leading to suppression of cell proliferation in hepatocellular carcinoma. Onco Targets Ther 2018;11:4451.

12. Wang J, Gao Y, Duan L, Wei S, Liu J, Tian L, et al. Metformin ameliorates skeletal muscle insulin resistance by inhibiting miR-21 expression in a high-fat dietary rat model. Oncotarget 2017;8(58):98029.

13. Zhang T, Li L, Shang Q, Lv C, Wang C, Su B. Circulating miR-126 is a potential biomarker to predict the onset of type 2 diabetes mellitus in susceptible individuals. Biochem Biophys Res Commun 2015;463(1-2):60-3.

14. Demirsoy IH, Ertural DY, Balci S, Cinkır U, Sezer K, Tamer $\mathrm{L}$, et al. Profiles of circulating miRNAs following metformin treatment in patients with type 2 diabetes. J Med Biochem 2018;37(4):499-506.

15. Den Dekker A, Davis FM, Kunkel SL, Gallagher KA. Targeting epigenetic mechanisms in diabetic wound healing. Transl Res 2019;204:39-50.

16. Qu Y, Wu J, Deng JX, Zhang YP, Liang WY, Jiang ZL, et al. MicroRNA-126 affects rheumatoid arthritis synovial fibroblast proliferation and apoptosis by targeting PIK3R2 and regulating PI3K-AKT signal pathway. Oncotarget 2016;7(45):74217.
17. Wu Y, Song LT, Li JS, Zhu DW, Jiang SY, Deng JY. MicroRNA-126 regulates inflammatory cytokine secretion in human gingival fibroblasts under high glucose via targeting tumor necrosis factor receptor associated factor 6 . J Periodontol 2017;88(11):e179-87.

18. Livak KJ, Schmittgen TD. Analysis of relative gene expression data using real-time quantitative PCR and the $2^{-\Delta \Delta C \mathrm{CT}}$ method. Methods 2001;25(4):402-8.

19. White R, McIntosh C. Topical therapies for diabetic foot ulcers: standard treatments. J Wound Care 2008;17(10):426-32.

20. Blakytny R, Jude E. The molecular biology of chronic wounds and delayed healing in diabetes. Diabet Med 2006;23(6):594608.

21. Wang Z, Li H, Guo R, Wang Q, Zhang D. Antioxidants inhibit advanced glycosylation end-product-induced apoptosis by downregulation of miR-223 in human adipose tissue-derived stem cells. Sci Rep 2016;6(1):1-11.

22. Alikhani Z, Alikhani M, Boyd CM, Nagao K, Trackman PC, Graves DT. Advanced glycation end products enhance expression of pro-apoptotic genes and stimulate fibroblast apoptosis through cytoplasmic and mitochondrial pathways. J Biol Chem 2005;280(13):12087-95.

23. Alikhani M, MacLellan CM, Raptis M, Vora S, Trackman PC, Graves DT. Advanced glycation end products induce apoptosis in fibroblasts through activation of ROS, MAP kinases and the FOXO1 transcription factor. Am J Physiol Cell Physiol 2007;292(2):C850-6.

24. Loughlin DT, Artlett CM. Precursor of advanced glycation end products mediates ER-stress-induced caspase-3 activation of human dermal fibroblasts through NAD (P) H oxidase 4. PLoS One 2010;5(6):e11093.

25. Ishibashi Y, Matsui T, Takeuchi M, Yamagishi S. Metformin inhibits advanced glycation end products (AGEs)-induced renal tubular cell injury by suppressing reactive oxygen species generation via reducing receptor for AGEs (RAGE) expression. Horm Metab Res 2012;44(12):891-5.

26. Gao J, Zhou XL, Kong RN, Ji LM, He LL, Zhao DB. MicroRNA-126 targeting PIK3R2 promotes rheumatoid arthritis synovial fibro-blasts proliferation and resistance to apoptosis by regulating PI3K/AKT pathway. Exp Mol Pathol 2016;100(1):192-8.

27. Li N, Tang A, Huang S, Li Z, Li X, Shen S, et al. MiR-126 suppresses colon cancer cell proliferation and invasion via inhibiting RhoA/ROCK signaling pathway. Mol Cell Biochem 2013;380(1):107-19.

This is an open access article distributed under the terms of the Creative Commons Attribution-NonCommercial-ShareAlike 3.0 License, which allows others to remix, tweak, and build upon the work non-commercially, as long as the author is credited and the new creations are licensed under the identical terms

This article was originally published in a special issue,
"Diagnostic and Therapeutic Advances in Biomedical Research
and Pharmaceutical Sciences"
Indian J Pharm Sci 2021:83(5) spl issue "69-74"

